\title{
The Extension Rate of the Medial Collateral Ligament of the Knee Joint during the Valgus Stress Test: Two Case Reports
}

\author{
Yuichi Takata1, Koji Iwamoto ${ }^{2 *}$, Sadanori Oshiro ${ }^{3}$, Mitsuhiro Iijima ${ }^{2}$ \\ ${ }^{1}$ Department of Physical Therapy, Faculty of Human Science, Hokkaido Bunkyo University, Hokkaido, Japan \\ ${ }^{2}$ Department of Physical Therapy, Ibaraki Prefectural University of Health Sciences, Ibaraki, Japan \\ ${ }^{3}$ Department of Physical Therapy, Okinawa College of Rehabilitation and Welfare, Okinawa, Japan \\ Email: ^iwamoto@ipu.ac.jp
}

How to cite this paper: Takata, Y., Iwamoto, K., Oshiro, S. and Iijima, M. (2021) The Extension Rate of the Medial Collateral Ligament of the Knee Joint during the Valgus Stress Test: Two Case Reports. Open Journal of Therapy and Rehabilitation, 9 , $1-9$.

https://doi.org/10.4236/ojtr.2021.91001

Received: December 17, 2020

Accepted: February 5, 2021

Published: February 8, 2021

Copyright $\odot 2021$ by author(s) and Scientific Research Publishing Inc. This work is licensed under the Creative Commons Attribution International License (CC BY 4.0).

http://creativecommons.org/licenses/by/4.0/

\begin{abstract}
This study aimed to evaluate the influence of measuring the length of the medial collateral ligament (MCL) to compare the MCL burden when the knee joint is placed under valgus stress in the open and closed and closed kinetic chain. Two examiners conducted the examination. The MCL length was measured using ultrasonography. Two subjects were measured in unload bearing and load-bearing positions, with and without valgus stress test at the knee joint extension and $30^{\circ}$ flexion, under eight different measurement conditions. The MCL of the subject was delineated in the longitudinal direction using an ultrasound system. The attachment points of the medial femoral and tibial condyle of the MCL were identified, and the ligament length was measured. The MCL rate before and after the valgus stress test in the loading and unloading positions was calculated. The MCL length increased by an average of $8.9 \%$ when the external stress test was performed in the non-weight bearing and knee extension positions and by an average of $17.0 \%$ when external stress was applied in the non-weight bearing and knee flexion positions. The MCL length increased by an average of $12.2 \%$ when the external stress test was performed in the load-bearing and knee extension positions and an average of $8.9 \%$ when the valgus stress test was applied in load-bearing and knee flexion positions. In conclusion, the effect of valgus stress on the MCL differs between load-bearing and non-load-bearing positions. It is considered that the dynamic stabilization mechanism works in the knee joint flexion position in the load position and works simultaneously as the static stabilization mechanism, which limits the knee joint valgus and reduces the extension rate of MCL. Therefore, this study reconsiders the shifting of traditional therapy from open kinetic chain to close kinetic chain.
\end{abstract}




\section{Keywords}

Medial Collateral Ligament, Valgus Stress Test, Extension Rate

\section{Introduction}

Of all knee ligament injuries, damage to the medial collateral ligament (MCL) is the most common ligamentous injury to the knee [1]. MCL injuries comprise up to $40 \%$ of all knee injuries [2]. In a 10-year observational study of athletes, it was reported that $7.9 \%$ of all the knee injuries consisted of MCL tears [3]. The medial support mechanism consists of dynamic and static stabilization systems. The dynamic stabilization system includes the vastus medialis, sartorius, gracilis, semimembranosus, and semitendinosus muscles.

The static stabilization system of the knee comprises the MCL and posterior oblique ligament (POL) [4].

The MCL is the most important tissue for the medial knee support mechanism and is divided into two layers: the superficial medial collateral ligament (sMCL) and the deep medial collateral ligament (dMCL) [5].

The sMCL, dMCL, and POL are the most commonly injured of the knee [6] [7] [8].

The injuries commonly result from situations in which valgus stress is applied to the knee and external rotation stress [9] [10] is applied to the lower leg with the foot fixed. It is often injured by tackling, as is often the case in soccer [11].

As the knee joint functions to provide support and drive, knee injuries require appropriate rehabilitation. Players returning to play in an imperfect condition are at risk of re-injury. Therefore, the conditions must be closely monitored.

In treating MCL injuries, it is important to understand the extent and condition of the damage correctly and undergo surgery as injury could easily recur if restoration of the tissue is incomplete.

Physical therapy for knee joint injuries generally begins with open kinetic chain (OKC) exercises before transitioning to closed kinetic chain (CKC) exercises. In the case of meniscus damage or osteoarthritis of the knee, in which the burden on the knee joint varies with load, OKC is first employed, followed by CKC.

Treatment of grade I and grade II MCL injuries is nonsurgical and focuses on early rehabilitation. Functional bracing using a hinged knee brace [12] [13] allows early range of motion (ROM) while protecting the knee from further valgus blows. ROM and weight-bearing are recommended as soon as the pain subsides [14].

However, a few studies have been conducted on the burden on the MCL in living bodies when the knee joint is in motion to compare differences between load-bearing and non-load-bearing positions and investigate their impact on the MCL. 
As differences in posture and position are expected to affect load in morphologically different ways, we consider it vital to proceed with a physiotherapy program to clarify these differences.

In this study, we measured the length of the MCL to compare the burden on the MCL when the knee joint is placed under valgus stress in load-bearing and non-load-bearing positions.

\section{Materials and Methods}

Before measurements and in accordance with the Declaration of Helsinki, we explained the details and purpose of this research to the participants both verbally and in writing and obtained their written consent to participate in this research. Subject characteristics are shown in Table 1. Our research consisted of two case reports.

A LOGIC BOOK-XP (GE Yokokawa Medical Systems) was used for ultrasonography, and an 8L-RS linear probe was employed. The ultrasonography apparatus was set to $B$ mode, the depth to $2 \mathrm{~cm}$, frequency to $11 \mathrm{~Hz}$, and gain and focus were adjusted for clear image depiction.

The examination was conducted by two examiners: one performed the ultrasonography, and the other manually applied valgus stress to the subject's knee. The flexion angle was set at $30^{\circ}$ [15], at which angle the knee is in a "loose" position, and valgus stress was applied by the examiner in the unload-bearing positions, although it was applied by the examinee himself in the load-bearing positions. The two examiners were physical therapy students who were taught to use the ultrasonograph by a physiotherapist with over 20 years of experience specializing in orthopedics physical therapy.

Subjects were measured in non-weight-bearing and weight-bearing positions, with and without valgus stress test at knee joint extension and $30^{\circ}$ of flexion, under eight different measurement conditions.

After the MCL of the subject was delineated in the longitudinal direction using an ultrasound system, a line was drawn on the contour of the MCL and the length was measured. The attachment points of the medial femoral and tibial condyle of the MCL were identified, and the length of the ligament was measured. The MCL rate before and after the valgus stress test in the loading and non-loading positions was calculated (Figures 1-8).

The measurement site was the posterior aspect of the MCL, confirmed at the medial side of the knee joint cleft by palpation.

Table 1. Subject characteristics.

\begin{tabular}{ccc}
\hline & Subject A & Subject B \\
\hline Sex & Male & Male \\
Age & 21 & 22 \\
Height $(\mathrm{m})$ & 1.73 & 1.71 \\
Weight $(\mathrm{kg})$ & 57 & 59.3 \\
BMI $\left(\mathrm{kg} / \mathrm{m}^{2}\right)$ & 19.0 & 20.3 \\
\hline
\end{tabular}




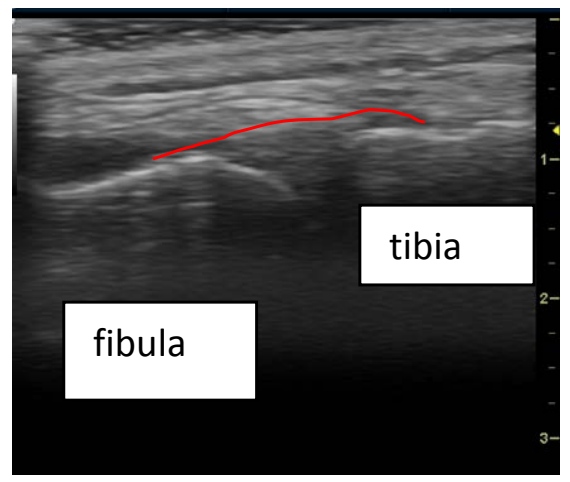

Figure 1. Knee extended unloaded unstressed.

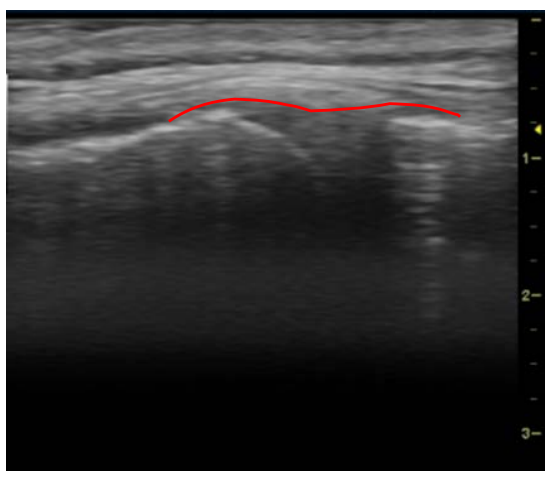

Figure 2. Knee extended unloaded stressed.

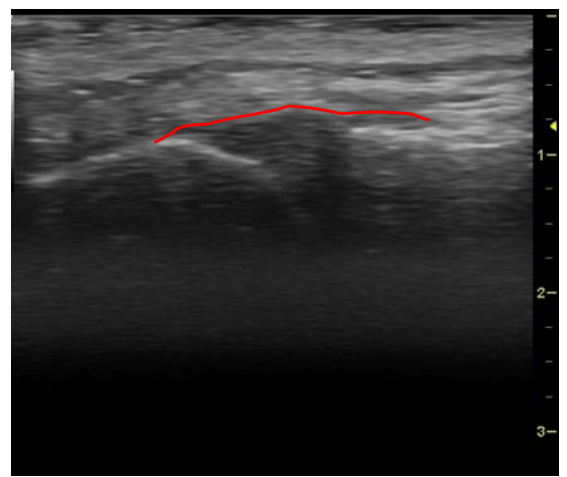

Figure 3. Knee extended loaded unstressed.

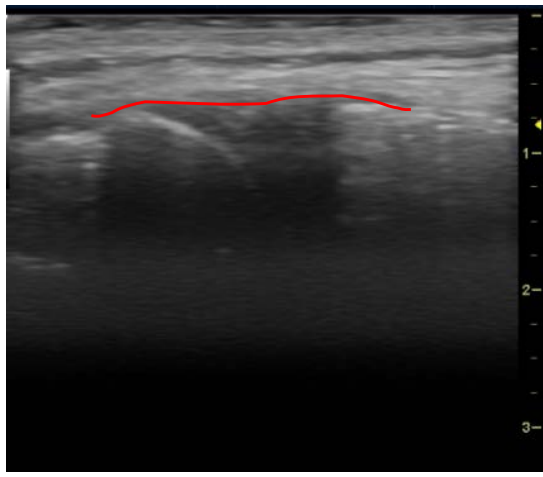

Figure 4. Knee extended loaded stressed. 


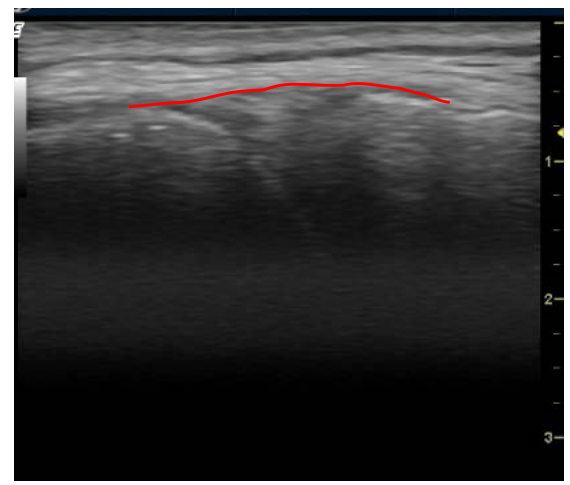

Figure 5. Knee flexed unloaded unstressed.

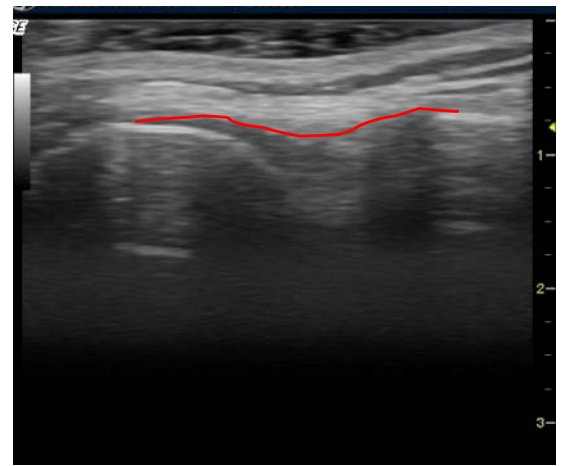

Figure 6. Knee flexed unloaded stressed.

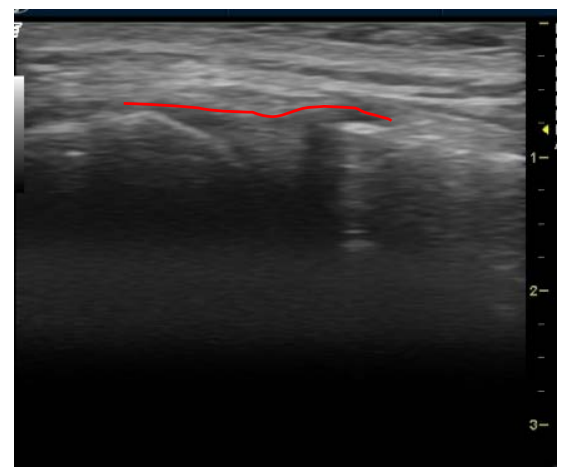

Figure 7. Knee flexed loaded unstressed.

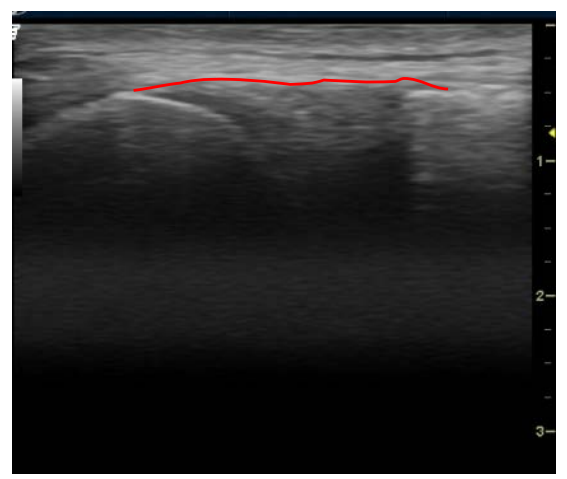

Figure 8. Knee flexed loaded stressed. 


\section{Results and Discussion}

Ultrasound images under valgus stress revealed that the knee cleft widens as the curve of the MCL straightens and confirms that the MCL tenses. In addition, when loaded, the knee cleft narrows, and we can confirm that the tension on the MCL slackens (Figures 1-8).

The results of this study are summarized in Table 2, while Table 3 shows the distance between attachments for each subject based on non-valgus stress values.

Table 3 shows that the length of the MCL increases by an average of $8.9 \%$ when the external stress test is performed in the non-weight bearing and knee extension positions and by an average of $17.0 \%$ when the external stress is applied to non-weight bearing and knee flexion positions.

In addition, the MCL length increases by an average of $12.2 \%$ when the external stress test is performed on the weight-bearing and knee extension positions and by an average of $8.9 \%$ when the valgus stress test is applied in the weight-bearing and knee flexion positions.

In the non-weight bearing position, the MCL lengthening rate during the external stress test was higher in the knee joint extension position than in the knee flexion position. In the loaded position, the MCL elongation rate was lower in the knee extension position than in the knee flexion position during the valgus stress test.

Table 2. MCL length $(\mathrm{cm})$ between the medial condyle of the femur and the medial condyle of the tibia, the attachment sites of the MCL, was measured.

\begin{tabular}{ccccccc}
\hline & Load & Knee Position & Valgus Stress & Subject A & Subject B & Average \\
\hline A & unload & Extended & - & 1.83 & 2.09 & 1.96 \\
B & unload & Extended & + & 1.93 & 2.35 & 2.14 \\
C & load & Extended & - & 2.27 & 2.24 & 2.26 \\
D & load & Extended & + & 2.59 & 2.47 & 2.53 \\
E & unload & flexed 30 & - & 2.26 & 2.36 & 2.31 \\
F & unload & flexed 30 & + & 2.52 & 2.89 & 2.71 \\
G & load & flexed 30 & - & 2.47 & 2.45 & 2.46 \\
H & load & flexed 30 & + & 2.71 & 2.65 & 2.68
\end{tabular}

Table 3. Amount of change in MCL before and after the valgus stress test (\%).

\begin{tabular}{cccccc}
\hline Load & Measurement Conditions & Knee Position & Subject A & Subject B & Average \\
\hline Unload & B-A & extended & 5.4 & 12.4 & 8.9 \\
Unload & F-E & flexed $30^{\circ}$ & 11.5 & 22.5 & 17.0 \\
Load & D-C & extended & 14.1 & 10.3 & 12.2 \\
Load & H-G & flexed 30 & 9.7 & 8.1 & 8.9 \\
\hline
\end{tabular}


In this study, we investigated the elongation rate of MCL in two healthy men when the knee joint was extended or the knee joint was flexed $30^{\circ}$ [15], and valgus stress tests were performed in the unloaded and loaded positions.

In the unloaded position, the MCL extension rate was high in the knee joint flexion position, and in the loaded position, the MCL extension rate was high in the knee joint extension position.

At the time of the valgus stress test in the unloaded position, it was reported that the stiffness of the MCL against valgus stress was stronger in the knee joint extension position than in the knee joint flexion position [16], which was consistent with the results of this study. It is considered that the ACL tension decreased in the knee extension position, and the MCL extension rate decreased in the knee extension position.

It has been reported that the lower limb muscles co-contract during lower limb exercises in a load position where the foot is in contact with the floor, such as a squat [17] [18].

Co-contraction of the quadriceps femoris and hamstrings [19], sartorius muscle [19], and gracilis muscle [20] has been reported as dynamic stabilization mechanisms, and it is considered that these muscle groups were active at the load position. It is considered that the dynamic stabilization mechanism works in the knee joint flexion position in the load position and works simultaneously as the static stabilization mechanism, which limits the knee joint valgus and reduces the extension rate of MCL.

Changing the exercise from the conventional OKC to CKC is considered appropriate for reducing the burden on MCL in physical therapy of MCL, but caution is required in the knee flexion position regardless of the load.

The limitations of this work are described as follows. Due to the small number of subjects in this study, statistical methods could not be used. In the future, it will be necessary to increase the number of subjects and verify the change in MCL elongation rate during knee valgus. In this study, we were able to capture a simple change in the length of the MCL between the loaded and unloaded positions, but we could not identify the location where the strain concentration occurred. This should be considered in future studies. This study has identified the changes in the length of the MCL when loaded and unloaded; however, we could not specify where the strain converged.

\section{Conflicts of Interest}

The authors declare no conflicts of interest regarding the publication of this paper.

\section{References}

[1] Andrews, K., Lu, A., McKean, L. and Ebraheim, N. (2017) Review: Medial Collateral Ligament Injuries. Journal of Orthopaedics, 14, 550-554. https://doi.org/10.1016/j.jor.2017.07.017

[2] Bollen, S. (2000) Epidemiology of Knee Injuries: Diagnosis and Triage. British 
Journal of Sports Medicine, 34, 227-228.

http://dx.doi.org/10.1136/bjsm.34.3.227-a

[3] Majewski, M., Susanne, H. and Klaus, S. (2006) Epidemiology of Athletic Knee Injuries: A 10-Year Study. Knee, 13, 184-188.

https://doi.org/10.1016/j.knee.2006.01.005

[4] Loredo, R., Hodler, J., Pedowitz, R., Yeh, L.R., Trudell, D. and Resnick, D. (1999) Posteromedial Corner of the Knee: MRI with Gross Anatomic Correlation. Skeletal Radiology, 28, 305-311. https://doi.org/10.1007/s002560050522

[5] LaPrade, R.F., Engebretsen, A.H., Ly, T.V., Johansen, S., Wentorf, F.A. and Engebretsen, L. (2007) The Anatomy of the Medial Part of the Knee. The Journal of Bone \& Joint Surgery, 89, 2000-2010. https://doi.org/10.2106/jbjs.f.01176

[6] Grood, E.S., Noyes, F.R., Butler, D.L. and Suntay, W.J. (1981) Ligamentous and Capsular Restraints Preventing Straight Medial and Lateral Laxity in Intact Human Cadaver Knees. The Journal of Bone \& Joint Surgery, 63, 1257-1269. https://doi.org/10.2106/00004623-198163080-00007

[7] Hughston, J.C. (1994) The Importance of the Posterior Oblique Ligament in Repairs of Acute Tears of the Medial Ligaments in Knees with and without an Associated Rupture of the Anterior Cruciate Ligament. Results of Long-Term Follow-up. The Journal of Bone \& Joint Surgery, 76, 1328-1344. https://doi.org/10.2106/00004623-199409000-00008

[8] Phisitkul, P., James, S.L., Wolf, B.R. and Amendola, A. (2006) MCL Injuries of the Knee: Current Concept Review. Iowa Orthopedic Journal, 26, 77-90.

[9] Laprade, R.F. and Wijdicks, C.A. (2012) Management of Injuries to the Medial Side of the Knee. Journal of Orthopaedic \& Sports Physical Therapy, 42, 221-233. https://www.jospt.org/doi/10.2519/jospt.2012.3624

[10] Wijdicks, C.A., Griffith, C.J., Johansen, S., Engebretsen, L. and LaPrade, R.F. (2010) Injuries to the Medial Collateral Ligament and Associated Medial Structures of the Knee. The Journal of Bone \& Joint Surgery, 92, 1266-1280. https://doi.org/10.2106/JBJS.I.01229

[11] Lundblad, M., Hägglund, M., Thomeé, C., Hamrin Senorski, E., Ekstrand, J., Karlsson, J., et al. (2019) Medial Collateral Ligament Injuries of the Knee in Male Professional Football Players: A Prospective Three-Season Study of 130 Cases from the UEFA Elite Club Injury Study. Knee Surgery, Sports Traumatology, Arthroscopy, 27, 3692-3698. https://doi.org/10.1007/s00167-019-05491-6

[12] Sandberg, R., Balkfors, B., Nilsson, B. and Westlin, N. (1987) Operative versus Non-Operative Treatment of Recent Injuries to the Ligaments of the Knee. A Prospective Randomized Study. The Journal of Bone \& Joint Surgery, 69, 1120-1126. https://doi.org/10.2106/00004623-198769080-00002

[13] Edson, C.J. (2006) Conservative and Postoperative Rehabilitation of Isolated and Combined Injuries of the Medial Collateral Ligament. Sports Medicine and Arthroscopy Review, 14, 105-110. https://doi.org/10.1097/01.jsa.0000212308.32076.f2

[14] Miyamoto, R.G., Bosco, J.A. and Sherman, O.H. (2009) Treatment of Medial Collateral Ligament Injuries. Journal of the American Academy of Orthopaedic Surgeons, 17, 152-161. https://doi.org/10.5435/00124635-200903000-00004

[15] Fetto, J.F. and Marshall, J.L. (1978) Medial Collateral Ligament Injuries of the Knee: A Rationale for Treatment. Clinical Orthopaedics and Related Research, No. 132, 206-218. https://doi.org/10.1097/00003086-197805000-00038

[16] Aronson, P.A., Rijke, A.M. and Ingersoll, C.D. (2008) Bilateral Medial Tibiofemoral Joint Stiffness in Full Extension and $20^{\circ}$ of Knee Flexion. Journal of Athletic Training, 43, 157-163. https://doi.org/10.4085/1062-6050-43.2.157 
[17] Baratta, R., Solomonow, M., Zhou, B.H., Letson, D., Chuinard, R. and D'Ambrosia R. (1988) Muscular Coactivation. The Role of the Antagonist Musculature in Maintaining Knee Stability. The American Journal of Sports Medicine, 16, 113-122. https://doi.org/10.1177/036354658801600205

[18] More, R.C., Karras, B.T., Neiman, R., Fritschy, D., Woo, S.L.-Y. and Daniel, D.M. (1993) Hamstrings: An Anterior Cruciate Ligament Protagonist. An in Vitro Study. The American Journal of Sports Medicine, 21, 231-237. https://doi.org/10.1177/036354659302100212

[19] Lloyd, D.G. and Buchanan, T.S. (2001) Strategies of Muscular Support of Varus and Valgus Isometric Loads at the Human Knee. Journal of Biomechanics, 34, 1257-1267. https://doi.org/10.1016/S0021-9290(01)00095-1

[20] Buchanan, T.S. and Lloyd, D.G. (1997) Muscle Activation at the Human Knee during Isometric Flexion-Extension and Varus-Valgus Loads. Journal of Orthopaedic Research, 15, 11-17. https://doi.org/10.1002/jor.1100150103 